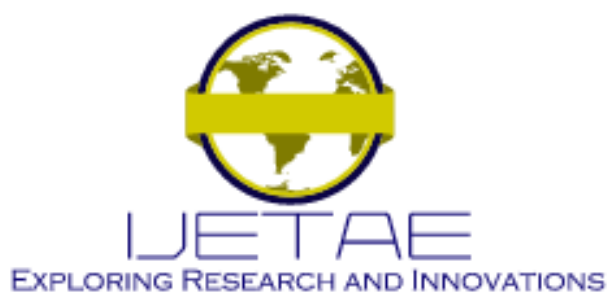

International Journal of Emerging Technology and Advanced Engineering

Website: www.ijetae.com (E-ISSN 2250-2459, Scopus Indexed, ISO 9001:2008 Certified Journal, Volume 12, Issue 01, January 2022)

Manuscript Received: 01 December 2021, Received in Revised form: 03 January 2022, Accepted: 10 January 2022

DOI: 10.46338/ijetae0122_02

\title{
Comparative Study of Forecasting Models for Forex Predictions with the Impact of Different Currencies
}

\author{
Satria Wiro Agung ${ }^{1}$, Kelvin Supranata Wangkasa Rianto ${ }^{2}$, Antoni Wibowo ${ }^{3}$ \\ ${ }^{1,2,3}$ Computer Science Department, BINUS Graduate Program - Master of Computer Science, Bina Nusantara University, \\ Jakarta, Indonesia, 11480
}

\begin{abstract}
Foreign Exchange (Forex) is the exchange / trading of currencies from different countries with the aim of making profit. Exchange rates on Forex markets are always changing and it is hard to predict. Many factors affect exchange rates of certain currency pairs like inflation rates, interest rates, government debt, term of trade, political stability of certain countries, recession and many more. Uncertainty in Forex prediction can be reduced with the help of technology by using machine learning. There are many machine learning methods that can be used when predicting Forex. The methods used in this paper are Long Short Term Memory (LSTM), Gated Recurrent Unit (GRU), Support Vector Regression (SVR). XGBOOST, and ARIMA. The outcome of this paper will be comparison results that show how other major currency pairs have influenced the performance and accuracy of different methods. From the results, it was proven that XGBoost outperformed other models by $0.36 \%$ compared to ARIMA model, $4.4 \%$ compared to GRU model, $8 \%$ compared to LSTM model, $9.74 \%$ compared to SVR model.
\end{abstract}

Keywords - Forex Forecasting, Long Short Term Memory, Gated Recurrent Unit, Support Vector Regression, ARIMA, Extreme Gradient Boosting

\section{INTRODUCTION}

Foreign Exchange (Forex) is the exchange / trading of currencies from different countries with the aim of making profit. Forex trading is different from manual transactions at money changers. Usually people who buy and sell money at money changers need to use a certain currency for the purpose like travelling and business. Whereas Forex aims to profit from currency exchange.

Exchange rates on Forex markets are always changing and it is hard to predict. This is a challenge that is faced by any trader whether they are beginner or professional.
Many factors affect exchange rates of certain currency pairs like inflation rates, interest rates, government debt, term of trade, political stability of certain countries, recession and many more. With these many factors trading Forex will be quite confusing and challenging for every trader especially for beginner traders.

Uncertainty in Forex prediction can be reduced with the help of technology. The technology we mention here is Machine Learning. With the help of Machine Learning we can create a system that can predict the exchange rate of a certain currency pair in the future. This Machine Learning system learns from previous exchange rate datas and mistakes of prediction from the past to improve themself over time.

There are many methods or models that can be used when predicting Forex. In [1] proposed a Forex prediction system that uses Linear Regression Line (LRL), and Dynamic Time Warping (DTW). Linear Regression Line is applied for analyzing and forming general trend patterns of historical Forex data of certain currency pairs. Trend patterns that analysed by Linear Regression Line will be learn by ANN. DTW is used to find similar trend patterns. CNN (Convolutional Neural Network) also can be used for prediction [2]. There are four convolutional layers in the proposed CNN model: CNN one-dimensional layer derived from Python keras library, one flatten layer and two fully connected layers. Convolutional operations perform convolved function which applies to input data with filters. Feature map is created using these filters. Flatten layer will generate a single dimensional feature matrix. [3] developed a model that is a combination of Convolution Neural Networks with Long Short Term Memory (CNN-LSTM). LSTM hidden layer, input layer, pooling layer, onedimensional convolution layer, and full connection layer make up the structure of the proposed model. For feature extraction, CNN model will be used. LSTM will be used for forecasting. 


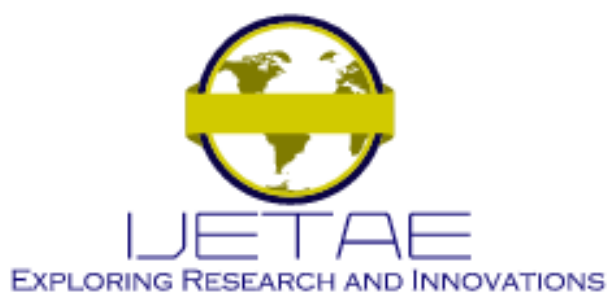

International Journal of Emerging Technology and Advanced Engineering Website: www.ijetae.com (E-ISSN 2250-2459, Scopus Indexed, ISO 9001:2008 Certified Journal, Volume 12, Issue 01, January 2022)

One of the problems that we found is most of the proposed models or methods by other author have not considered the influence of other factors (such as commodities, inflation, public debt, other currencies etc.) than the predicted currency pair data (open, close, high, low, etc). Therefore in this paper, comparison of the existing methods with influence of other major currency pairs will be conducted. In this paper, the currency pair USD/EUR will be predicted with the influence of other major currency pairs (USD/GBP and USD/JPY). LSTM, GRU, SVR, XGBoost, and ARIMA are the methods used in this paper. The outcome of this paper will be comparison results that show how other major currency pairs have influence the performance and accuracy of different models or methods.

\section{RELATED WORKS}

The methods that are used in stock prediction can also be used for Forex prediction because of the similarity of the market's behavior. [1] proposed a prediction system that uses Linear Regression Line (LRL) for segmentation, ANN for classification, and Dynamic Time Warping (DTW) for recognition. Linear Regression Line is applied for analyzing and forming general trend patterns of historical Forex data of certain currency pairs. Trend patterns that analysed by Linear Regression Line will be learn by ANN. DTW is used to find similar trend patterns. The result of the proposed system is evaluated by comparing the accuracy of the proposed system with other approaches that use only normal ANN and Support Vector Machine (SVM). The evaluated result shows that the proposed system has better accuracy than other approaches. The proposed system has 72 percent accuracy. Other approaches like ANN has an accuracy of 59 percent, and SVM has an accuracy of 70 percent.

[2] proposed a CNN model to predict stock market prices. There are four convolutional layers in the proposed CNN model: CNN one-dimensional layer derived from Python keras library, one flatten laCyer and two fully connected layers. Convolutional operations perform convolved function which applies to input data with filters. Feature map is created using these filters. Flatten layer will generate a single dimensional feature matrix. The evaluation is conducted by comparing error rate (RMSE) for various epochs (10, 20, 40, 60, 100). After 100 epoch, the RMSE is 1.21 better than the first ten epoch which RMSE is 9.76 .
[3] proposed a stock prediction model that combines Convolution Neural Network with Long Short Term Memory (CNN-LSTM). For feature extraction, CNN model will be used. LSTM will be use for forecasting. The evaluation is conducted by comparing the performance of other models like CNN, RNN, CNN-RNN, CNN-LSTM with multilayer perceptron (MLP), and LSTM. From the results, it can be seen that CNN-LSTM has the lowest MAE and RMSE (27.564 and 39.688) compared to others. CNN-LSTM has the highest R2 score (0.9646). From the result it can be concluded that CNN-LSTM has better accuracy.

[4] proposed a model that combines Piecewise Linear representation (PLR) and Neural Network for stock prediction. PLR is used in data processing, where PLR decomposes time series data into a set of disjoint line segments to generate smoother trends of the input variables. Then ANN is used for stock prediction. ANN model consists of four input neurons on input layer, four hidden neurons on hidden layer and one output neuron on output layer. The result of this experiment is not optimal because the average prediction result is about $54.62 \%$.

[5] proposed a combination of ARIMA and GRU models for stock prediction. ARIMA models take opening price data as input. Then the output of the ARIMA model will be used as input variables for the GRU model. The results are evaluated by comparing the result of the ARIMA-BPNN model with the proposed solution ARIMA-GRU model. The result of the experiment shows a big difference between the ARIMA-BPNN and ARIMA-GRU model, according to the graph where the ARIMA-BPNN model has the worst prediction accuracy and the ARIMA-GRU model has the best prediction accuracy.

[6] proposed a prediction model for stock forecasting that combines Particle Swarm Optimization (PSO) with Least Square Support Vector Machine (LS-SVM). LSSVM will be optimized using PSO algorithm. The proposed model will use six input: the historical data and derived technical indicators (Exponential Moving Average, Stochastic Oscillator, Relative Strength Index, Money Flow Index, Moving Average Convergence) and one output represents next price. The evaluation is conducted by comparing Mean Square Error between results of single LS-SVM, PSO-LSSVM, and Neural Network Back Propagation method. From the graphic results, can be seen the proposed model (PSO-LSSVM) has the lowest error value followed by single LS-SVM and NN-BP algorithm is the worst one. 


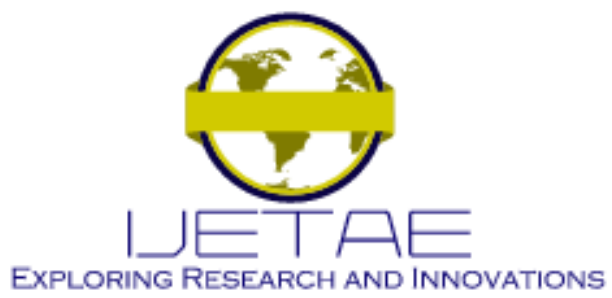

International Journal of Emerging Technology and Advanced Engineering Website: www.ijetae.com (E-ISSN 2250-2459, Scopus Indexed, ISO 9001:2008 Certified Journal, Volume 12, Issue 01, January 2022)

[7] conducted research to improve predictions of currency exchange rates using the ARIMA model by adding a hybrid model of the Multilayer Perceptron (MLP) and Radial Basis Function (RBF). The added hybrid model will be used as error modeling to improve the final outcome of the prediction. The data used in this research is the exchange rate of the Euro against the US Dollar. The final results of this study indicate that the proposed model has better result than the single model with the values (RMSE, D-stat) of the hybrid model, single ARIMA, single MLP and single RBF respectively $(0.0068,71 \%),(0.0081$, $65 \%),(0.0076,67 \%),(0.0085,59 \%)$.

[8] conducted research by proposing the ARI-MA-LSSVM (Auto Regressive Integrated Moving Average - Least Square - Support Vector Machine) hybrid model to predict stock value. The method of this model is to enter raw data into the ARIMA model, from the ARIMA model 2 values will be generated, namely low frequency information and high frequency information. Low frequency information will be re-entered as input into the SVM model while High frequency information will be entered as input into the LSSVM model. Then the results of the predictions of the two SVM models will be synthesized to produce the final predictions. The results of the model will then be compared with the results of other models such as LS-SVM and RSSVM based on the MSE and RMSE values. The results showed that combination of ARIMA with LS-SVM had a better result than the hybrid LS-SVM and hybrid RS-SVM models with the MSE and RMSE values of 0.015 and 0.108 , respectively.

[9] conducted a study to predict the stock value of Dhaka using the ANN and RS (Rough Set) hybrid method. The results of this proposed hybrid model are compared for the single RS and ANN models. The performance of each model will be seen using a confusion matrix and its accuracy value. The experimental results show that the proposed hybrid model has an accuracy of $97 \%$ which is higher than the single RS and ANN models.
[10] conducted research by evaluating the performance resulting from the Neural Network, Vector Singular Spectrum Analysis (VSSA) and Recurrent Singular Spectrum Analysis (RSSA) against currency exchange rates from the UK, Europe and China. The parameter used to evaluate these models is to use the model accuracy level. From these studies, it was found that the SSA model has an accuracy of $67 \%$ better than the usual NN model.

Research using several models such as the Nonlinear Ensemble (NE), Generalized Linear Auto Regression (GLAR), ANN, and Hybrid GLAR-ANN was conducted by [11]. This research uses data on the German Marks, British Pound and Japanese Yen in making predictions. The performance of the model used will be measured using the NMSE value, D-stat and the percentage of the annual return rate. From the results of this study it can be concluded that the nonlinear ensemble model has the best performance compared to the other models studied because it has the lowest NMSE value and D-stat and the high percentage of annual return rates.

[12] conducted a similar study in predicting forex movements using the hybrid machine learning concept that combines 2 regression models, namely the LSTM and GRU. The research was conducted on several forex data, namely EUR / USD, GBP / USD, USD / CAD and USD / CHF. The parameters used in this study are open price, high price, low price, close price, momentum, average price, range, and also OLHC price with predicted data intervals of 10 minutes and 30 minutes. The performance of the proposed model will be compared with several general models such as LTSM, GRU and SMA using assessments based on MSE, RMSE and MAE scores. The results show that the proposed hybrid model has better performance than a single algorithm with the mean values of MSE, RMSE, and MAE, respectively, 0.000035, 0.00404 and 0.00281 . 


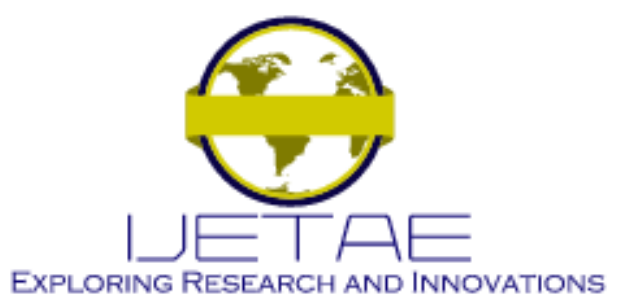

International Journal of Emerging Technology and Advanced Engineering

Website: www.jjetae.com (E-ISSN 2250-2459, Scopus Indexed, ISO 9001:2008 Certified Journal, Volume 12, Issue 01, January 2022)

TABLE 1

RELATED WORKS SUMMARY

\begin{tabular}{|c|c|c|c|}
\hline Source & $\begin{array}{l}\text { Domain } \\
\text { Problem }\end{array}$ & Methods & Results \\
\hline$[1]$ & $\begin{array}{l}\text { Foreign } \\
\text { Exchange } \\
\text { Prediction }\end{array}$ & $\begin{array}{l}\text { Hybrid LRL - } \\
\text { ANN - DTW }\end{array}$ & $\begin{array}{l}\text { The proposed system has } \\
72 \text { percent accuracy. }\end{array}$ \\
\hline$[2]$ & \begin{tabular}{|l} 
Stock \\
Prediction
\end{tabular} & $\mathrm{CNN}$ & $\begin{array}{l}\text { The proposed method } \\
\text { has RMSE of } 1.21 .\end{array}$ \\
\hline [3] & $\begin{array}{l}\text { Stock } \\
\text { Prediction }\end{array}$ & $\begin{array}{l}\text { Hybrid } \\
\text { LSTM }\end{array}$ & $\begin{array}{l}\text { CNN-LSTM has the } \\
\text { lowest MAE and RMSE } \\
\text { (27.564 and 39.688). }\end{array}$ \\
\hline [4] & $\begin{array}{l}\text { Stock } \\
\text { Prediction }\end{array}$ & $\begin{array}{l}\text { Hybrid PLR } \\
\text { ANN }\end{array}$ & $\begin{array}{l}\text { The result of this } \\
\text { experiment is not } \\
\text { optimal because the } \\
\text { average prediction result } \\
\text { is about } 54.62 \% \text {. }\end{array}$ \\
\hline$[5]$ & $\begin{array}{l}\text { Stock } \\
\text { Prediction }\end{array}$ & $\begin{array}{l}\text { Hybrid ARIMA - } \\
\text { GRU }\end{array}$ & $\begin{array}{l}\text { ARIMA-GRU model has } \\
\text { the best prediction } \\
\text { accuracy. }\end{array}$ \\
\hline$[6]$ & $\begin{array}{l}\text { Stock } \\
\text { Prediction }\end{array}$ & $\begin{array}{l}\text { Hybrid PSO - LS } \\
\text { - SVM }\end{array}$ & $\begin{array}{l}\text { The proposed model } \\
\text { (PSO-LS-SVM) has the } \\
\text { lowest error value. }\end{array}$ \\
\hline [7] & $\begin{array}{l}\text { Foreign } \\
\text { Exchange } \\
\text { Prediction }\end{array}$ & $\begin{array}{l}\text { Hybrid ARIMA - } \\
\text { MLP - RBF }\end{array}$ & $\begin{array}{l}\text { Proposed method Hybrid } \\
\text { ARIMA - MLP - RBF } \\
\text { has better result than the } \\
\text { single model with the } \\
\text { RMSE and D-stat values } \\
\text { of } 0.0068 \text { and } 71 \% \text {, } \\
\text { respectively. }\end{array}$ \\
\hline [8] & $\begin{array}{l}\text { Stock } \\
\text { Prediction }\end{array}$ & $\begin{array}{l}\text { Hybrid ARIMA - } \\
\text { LS - SVM }\end{array}$ & $\begin{array}{l}\text { Proposed method hybrid } \\
\text { ARIMA - LS - SVM has } \\
\text { better result than the } \\
\text { hybrid LS-SVM and RS- } \\
\text { SVM with the MSE and } \\
\text { RMSE values of } 0.015 \\
\text { and } 0.108 \text {, respectively. }\end{array}$ \\
\hline [9] & $\begin{array}{l}\text { Stock } \\
\text { Prediction }\end{array}$ & Hybrid ANN - RS & $\begin{array}{l}\text { The experimental results } \\
\text { show that the proposed } \\
\text { hybrid model has an } \\
\text { accuracy of } 97 \% \text { which } \\
\text { is higher than the single } \\
\text { RS and ANN models. }\end{array}$ \\
\hline [10] & $\begin{array}{l}\text { Foreign } \\
\text { Exchange } \\
\text { Prediction }\end{array}$ & SSA & $\begin{array}{l}\text { SSA model has an } \\
\text { accuracy of } 67 \% \text { better } \\
\text { than the usual NN } \\
\text { model. }\end{array}$ \\
\hline [11] & $\begin{array}{l}\text { Foreign } \\
\text { Exchange }\end{array}$ & \begin{tabular}{|r} 
NE, GLAR, ANN \\
and
\end{tabular} & $\begin{array}{l}\text { The nonlinear ensemble } \\
\text { model has the best }\end{array}$ \\
\hline
\end{tabular}

\begin{tabular}{|c|c|c|c|}
\hline & Prediction & GLAR-ANN & $\begin{array}{l}\text { performance compared } \\
\text { to the other models } \\
\text { studied because it has the } \\
\text { lowest NMSE value and } \\
\text { D-stat and the high } \\
\text { percentage of annual } \\
\text { return rates. }\end{array}$ \\
\hline [12] & $\begin{array}{l}\text { Foreign } \\
\text { Exchange } \\
\text { Prediction }\end{array}$ & $\begin{array}{l}\text { Hybrid LSTM } \\
\text { GRU }\end{array}$ & $\begin{array}{l}\text { Proposed method hybrid } \\
\text { LSTM - GRU has better } \\
\text { performance than a } \\
\text { single algorithm with the } \\
\text { mean values of MSE, } \\
\text { RMSE, and MAE, } \\
\text { respectively, 0.000035, } \\
0.00404 \text { and } 0.00281 .\end{array}$ \\
\hline
\end{tabular}

\section{RESEARCH METHODOLOGY}

\section{A. Data Acquisition}

The main objective of this research is to compare various prediction methods and to analyze the effect of currency rate movements between one another. The dataset used in this study will be collected from investing website [13] with a period from 2010 to 2020. Data was collected for three major currency pairs: USD/EUR [14], USD/GBP [15], and USD/JPY [16]. For each dataset contains a total of 5 attributes: Date and time, High price, Low price, Open price, and Close price.

\section{B. Data Preprocessing}

In the preprocessing stage, a new feature will be created for each currency exchange rate with the following calculations:

$$
\begin{gathered}
\text { Momentum }=\text { close price }- \text { open price } \#(1) \\
\text { Average price }=\frac{(\text { high price }- \text { low price })}{2} \#(2) \\
\text { Range }=\text { high price }- \text { low price } \#(3) \\
O H L C=\frac{(\text { open price }+ \text { high price }+ \text { low price }+ \text { close price })}{4} \#(4)
\end{gathered}
$$

After the new feature is created, 3 dataset groups will be formed which are a combination of features from one or more currency exchange rates.

Detailed features for each dataset group are shown in Table 1. 


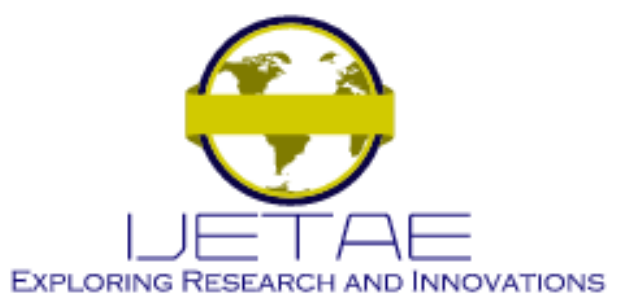

International Journal of Emerging Technology and Advanced Engineering

Website: www.ijetae.com (E-ISSN 2250-2459, Scopus Indexed, ISO 9001:2008 Certified Journal, Volume 12, Issue 01, January 2022)

TABLE 2

DETAILED FEATURES FOR EACH DATASET GROUP

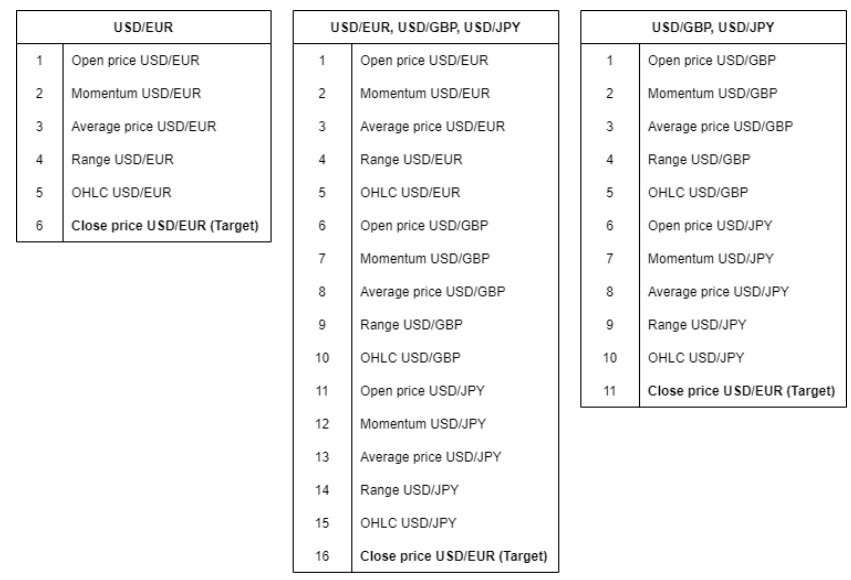

\section{Long Short Term Memory}

LSTM (Long Short Term Memory) was proposed by [17]. The conventional recurrent neural network has problems where error signals or gradients when backpropagation tend to either blow up or vanish. LSTM uses memory cells and gates to keep information for long periods and to forget unnecessary information. LSTM consists of a memory cell and three gates : forget gate, output gate, and input gate [18].

The purpose of the input gate is to determine what information should be kept and what information should be updated. To decide which information should be updated, current input and previous hidden state are passed into a sigmoid function. Which the values will be updated by transforming it into the values between 0 and 1. Zero (0) means not important and 1 means important. The forget gate uses sigmoid function to determine what information should be forgotten. Next hidden state is decided by the output gate and the hidden state is then carried over to the next time step.

The LSTM has two phases: The forward pass and the backpropagation[19]. In the forward pass, the weights of LSTM can be categorized as:

- Input weights : $W_{z}, W_{i}, W_{f}, W_{o} \in R^{N * M}$

- Recurrent weights: $R_{z}, R_{i}, R_{f}, R_{o} \in R^{N * N}$

- Peephole weights: $p_{i}, p_{f}, p_{o} \in R^{N}$

- Bias weights: $b_{z}, b_{i}, b_{f}, b_{o} \in R^{N}$
Where:

- $\quad z$ : block input

- $\quad i$ : input gate

- $f:$ forget gate

- $o$ : output gate

- $\quad N$ : number of LSTM blocks

- $\quad M \quad$ : number of inputs

The formulation of forward pass in Vanilla LSTM can be defined as below [19] :

$$
\begin{gathered}
\bar{z}^{t}=W_{z} x^{t}+R_{z} y^{t-1}+b_{z} \#(5) \\
z^{t}=g\left(\bar{z}^{t}\right) \# \text { block input }(6) \\
\bar{l}^{t}=W_{i} x^{t}+R_{i} y^{t-1}+p_{i} \odot c^{t-1}+b_{i} \#(7) \\
i^{t}=\sigma\left(\bar{l}^{t}\right) \# \text { input gate }(8) \\
\bar{f}^{t}=W_{f} x^{t}+R_{f} y^{t-1}+p_{f} \odot c^{t-1}+b_{f} \#(9) \\
f^{t}=\sigma\left(\bar{f}^{t}\right) \# \text { forget gate (10) } \\
c^{t}=z_{t} \odot i_{t}+c^{t-1} \odot f^{t} \# \text { cell (11) } \\
\bar{o}^{t}=W_{o} x^{t}+R_{o} y^{t-1}+p_{o} \odot c^{t}+b_{o} \#(12) \\
o^{t}=\sigma\left(\bar{o}^{t}\right) \# \text { \#utput gate }(13) \\
y^{t}=h\left(c^{t}\right) \odot o^{t} \# \text { block output }
\end{gathered}
$$

Where:

- $x^{t}$ : input vector

- $y^{t}$ : block output

- $\quad c^{t}$ : cell at time $\mathrm{t}$

- $\sigma \quad$ : logistic sigmoid function

- $g \quad$ : hyperbolic tangent functions

- $h \quad$ : hyperbolic tangent functions

- $\odot$ : point-wise multiplication of the the two vectors

In the backpropagation, deltas of LSTM blocks and gradient of the weights will be calculated [19]. The equation for calculating deltas of LSTM blocks are shows as below:

$$
\begin{gathered}
\delta y^{t}=\Delta^{t}+R_{z}{ }^{T} \delta z^{t+1}+R_{i}{ }^{T} \delta i^{t+1}+R_{f}{ }^{T} \delta f^{t+1}+R_{o}{ }^{T} \delta o^{t+1} \#(15) \\
\delta \bar{o}^{t}=\delta y^{t} \odot h\left(c^{t}\right) \odot \sigma^{\prime}\left(\bar{o}^{t}\right) \#(16) \\
\delta c^{t}=\delta y^{t} \odot o^{t} \odot h^{\prime}\left(c^{t}\right)+p_{o} \odot \delta \bar{o}^{t}+p_{i} \odot \delta \bar{t}^{t+1} \\
+p_{f} \odot \delta \bar{f}^{t+1}+\delta c^{t+1} \odot f^{t+1} \#(17) \\
\delta \bar{f}^{t}=\delta c^{t} \odot c^{t-1} \odot \sigma^{\prime}\left(\bar{f}^{t}\right) \#(18) \\
\delta \overline{\mathbf{l}}^{\mathrm{t}}=\delta c^{\mathrm{t}} \odot \mathrm{z}^{\mathrm{t}} \odot \sigma^{\prime}\left(\overline{\mathrm{i}}^{\mathrm{t}}\right) \#(19)
\end{gathered}
$$




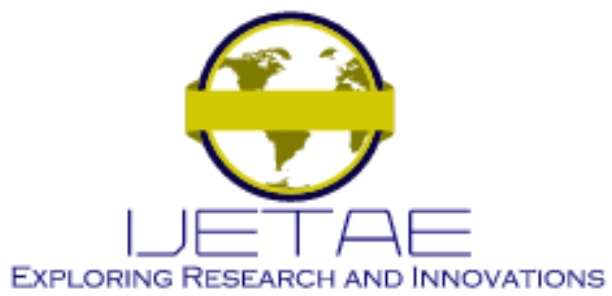

\section{International Journal of Emerging Technology and Advanced Engineering}

Website: www.ijetae.com (E-ISSN 2250-2459, Scopus Indexed, ISO 9001:2008 Certified Journal, Volume 12, Issue 01, January 2022)

$$
\begin{gathered}
\delta \bar{z}^{t}=\delta c^{t} \odot i^{t} \odot g^{\prime}\left(\bar{z}^{t}\right) \#(20) \\
\delta x^{t}=W_{z}{ }^{T} \delta \bar{z}^{t}+W_{i}{ }^{T} \delta \bar{\imath}^{t}+W_{f}{ }^{T} \delta \bar{f}^{t}+W_{o}{ }^{T} \delta \bar{o}^{t} \#(21)
\end{gathered}
$$

Where:

- $\quad \Delta^{t}:$ vector of the deltas

- $\quad T$ : transposition operator

Following equations is for calculate the gradient of the weights:

$$
\begin{aligned}
\delta \mathrm{W}_{*} & =\sum_{t=0}^{T}<\delta *^{\mathrm{t}}, \mathrm{x}^{\mathrm{t}}>\#(22) \\
\delta \mathrm{R}_{*} & =\sum_{t=0}^{T-1}<\delta *^{\mathrm{t}+1}, \mathrm{y}^{\mathrm{t}}>\#(23) \\
\delta \mathrm{b}_{*} & =\sum_{t=0}^{T} \delta * \mathrm{t} \#(24) \\
\delta \mathrm{p}_{\mathrm{i}} & =\sum_{t=0}^{T-1} \mathrm{c}^{\mathrm{t}} \odot \delta \overline{\mathrm{r}}^{\mathrm{t}+1} \#(25) \\
\delta \mathrm{pf} & =\sum_{t=0}^{T-1} \mathrm{c}^{\mathrm{t}} \odot \delta \overline{\mathrm{f}}^{\mathrm{t}+1} \#(26) \\
\delta \mathrm{po} & =\sum_{t=0}^{T} \mathrm{c}^{\mathrm{t}} \odot \delta \overline{\mathrm{o}}^{\mathrm{t}} \#(27)
\end{aligned}
$$

Where:

$$
\begin{aligned}
& \text { - } \quad * \quad \text { : can be any of }\{\bar{z}, \bar{i}, \bar{f}, \bar{o}\} \\
& \text { - }<*_{1}, *_{2}>\quad \text { : outer product derived from the two } \\
& \text { vectors } \\
& \text { - } \mathrm{T} \quad \text { : vector length }
\end{aligned}
$$

\section{Gated Recurrent Unit}

Gated Recurrent Unit (GRU) is proposed by [20]. GRU is a variant of recurrent neural networks. GRU is simpler than LSTM and GRU only contains two gates (update gate and reset gate). [21] Update gate control past informations that will be passed. Reset gate controls what past information will be forget. Sigmoid activation function will be use in update gate and reset gate. The following are equations that used in GRU operation [5,20,22]:

$$
\begin{gathered}
R_{t}=\sigma\left(X_{t} W_{x r}+H_{t-1} W_{h r}+b_{r}\right) \#(28) \\
Z_{t}=\sigma\left(X_{t} W_{x z}+H_{t-1} W_{h z}+b_{z}\right) \#(29) \\
\bar{H}_{t}=\tanh \left(X_{t} W_{x h}+\left(R_{t} \odot H_{t-1}\right) W_{h h}+b_{h}\right) \#(30) \\
H_{t}=Z_{t} \odot H_{t-1}+\left(1-Z_{t}\right) \odot \bar{H}_{t} \#(31)
\end{gathered}
$$

Where:

$$
\begin{array}{ll}
\text { - } & W \quad \text { : Weight } \\
\text { - } & X \quad \text { : Input } \\
\text { - } & b: \text { Bias } \\
\text { - } & R_{t} \quad: \text { Reset gate } \\
\text { - } & Z_{t} \quad: \text { Update gate } \\
\text { - } & \bar{H}_{t} \quad \text { : Candidate activation } \\
\text { - } & H_{t} \quad \text { : Actual activiation }
\end{array}
$$

\section{E. Support Vector Regression}

Support Vector Regression (SVR) is a regression model that based on implementation of Support Vector Machine (SVM). Usually, SVM is used for solving classification problems but with addition of several parameters SVM can be used for solving regression problems. SVR have additional parameters like $\varepsilon$ (epsilon) and slack $(\xi)$. Epsilon is the width of around the hyperplane. The predictions or point that fall inside this width are considered as correct prediction and it is assumed that there is no prediction error [23]. Slack is the distance between the epsilon and points outside the hyperplane. Slack determines how much tolerance for points outside the epsilon. The purpose of $\mathrm{SVR}$ is to find the best linear function $\mathrm{f}$ which provides the best approximation of the actual output $y$ with an error tolerance $\varepsilon$ and is concurrently as flat as possible [24]. Suppose there is input $\mathrm{S}=\left\{\left(\mathrm{x}_{1}, \mathrm{y}_{1}\right),\left(\mathrm{x}_{2}, \mathrm{y}_{2}\right), \ldots,\left(\mathrm{x}_{\mathrm{i}}, \mathrm{y}_{\mathrm{i}}\right)\right\}$. Relationship between output and input is approximates with linear function as belows:

$$
f(x, w)=(w \cdot x+b) \#(32)
$$

But in the real-world application, input data is not always linearly separable. This is where the kernel function is used. Kernel function / nonlinear mapping function $\phi(\mathrm{x})$ mapping input space to another higher dimension feature space [23]. Higher dimension transformation is needed to make linear separation possible.

$$
f(x, w)=(w \cdot \phi(x)+b) \#(33)
$$




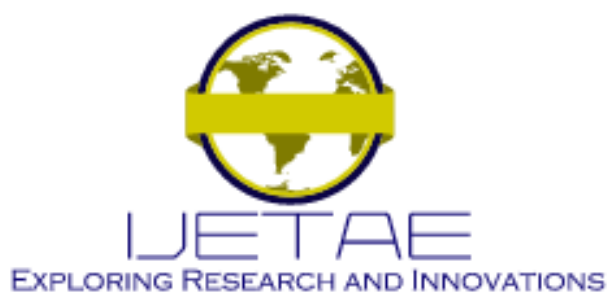

International Journal of Emerging Technology and Advanced Engineering Website: www.ijetae.com (E-ISSN 2250-2459, Scopus Indexed, ISO 9001:2008 Certified Journal, Volume 12, Issue 01, January 2022)

The purpose of SVR is to minimizing the following regularized functions [25]:

$$
\begin{gathered}
\text { Minimize: } R(f)=C \frac{1}{N} L_{\varepsilon}(y, f(x, w))+\frac{1}{2}\left\|W^{2}\right\| \#(34) \\
L_{\varepsilon}(y, f(x, w))=f(x)=\left\{\begin{array}{cc}
0, \quad \text { if }|y-f(x, w)| \leq \varepsilon \\
|y-f(x, w)|-\varepsilon, \quad \text { otherwise }
\end{array}\right.
\end{gathered}
$$

But if slack variables $\xi$ and $\xi^{*}$ is presented then the purpose of SVR is to minimize following convex optimization problem:

$$
\text { Minimize: } \frac{1}{2}\left\|W^{2}\right\|+C \sum_{i=1}^{N} \xi_{i}+\xi_{i}^{*} \#(36)
$$

Subject to

$$
\begin{gathered}
\left(y_{i}-w \cdot \phi(x)-b\right) \leq \varepsilon+\xi_{i} \\
\left(w \cdot \phi(x)+b-y_{i}\right) \leq \varepsilon+\xi_{i}^{*}, \#(38) \\
\xi_{i}, \xi_{i}^{*} \geq 0 \#(39)
\end{gathered}
$$

\section{F. Auto Regressive Integrated Moving Average}

ARIMA or Auto Regressive Integrated Moving Average as the name implies consists of 3 different models, Autoregressive (AR) model, Integrated (I) model and Moving Average Model (MA). Autoregressive model is a model that can predict a value based on past values. This model assumes that there is a correlation between present values and past values. The term moving average refers to a model that can smooth out values within a certain range. Moving Averages are usually used to remove noise or random movements in the data. In addition, the use of Moving Average also shows that the predicted value depends linearly on the previous values. Meanwhile, the Integrated model refers to the model used to convert nonstationary data into stationary by applying differencing to the data. This Integrated model will eliminate the existing trend in the data.

For operating the ARIMA model by the Box-Jenkins methodology, there are three steps that should be considered : identification, estimation of parameters and forecasting [26]. The identification step is done by checking whether the dataset used is stationary.
If the dataset used is not stationary, differencing will be performed until the data becomes stationary. Estimating the model parameters can be done by using auto correlation function (ACF) and partial ACF (PACF). The identification step is done by checking whether the dataset used is stationary. If the dataset used is not stationary, differencing will be performed until the data becomes stationary. Estimating the model parameters can be done by using auto correlation function (ACF) and partial ACF (PACF). Parameters in ARIMA model can be classified into 3 terms (p, q, d) where: $p$ is the number of autoregressive (AR), $d$ is the number of differencing until the dataset becomes stationary (I), and $\mathrm{q}$ is the window size used in the Moving Average (MA). The formula used by the ARIMA model to predict can be defined as follows [5]:

$$
\begin{gathered}
(1-B) Y_{t}=\delta+\varphi_{1} Y_{t-1}+\varphi_{2} Y_{t-2}+\varphi_{3} Y_{t-3}+\ldots+\varphi_{p} Y_{t-p} \\
\quad+\varepsilon_{t}-\varepsilon_{t-1} \theta_{1}-\varepsilon_{t-2} \theta_{2}-\varepsilon_{t-3} \theta_{3}+\ldots+\varepsilon_{t-q} \theta_{q} \#(40)
\end{gathered}
$$

Where:

$$
\begin{array}{ll}
\mathrm{B} & : \text { backshift operator } \\
\mathrm{Y}_{\mathrm{t}} & : \text { value at time } \mathrm{t} \\
\delta & : \text { bias } \\
\varphi & : \text { auto regressive parameter } \\
\varepsilon_{\mathrm{t}} & : \text { random error at time } \mathrm{t} \\
\theta & : \text { moving average parameter }
\end{array}
$$

\section{G. XGBoost}

Extreme Gradient Boosting or commonly known as XGBoost is an implementation of a boosting algorithm that uses multiple decision trees. The term "gradient boosting" in XGBoost refers to the use of the gradient descent algorithm to minimize the loss function generated by each decision tree [27]. While the term "extreme" refers to the goal of XGBoost itself to maximize the computation of the gradient boosting machine algorithm by implementing various enhancements and optimizations. Some of the advantages that XGBoost has compared to ordinary gradient boosting include: regularization to reduce overfitting, parallel processing which can process data faster, efficiently handling missing data using the sparse approach, cache awareness to increase performance and others. The process of making predictions using the XGBOOST model can be seen in Figure 1 


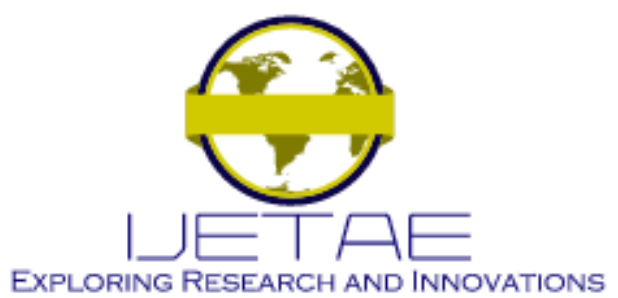

International Journal of Emerging Technology and Advanced Engineering

Website: www.ijetae.com (E-ISSN 2250-2459, Scopus Indexed, ISO 9001:2008 Certified Journal, Volume 12, Issue 01, January 2022)

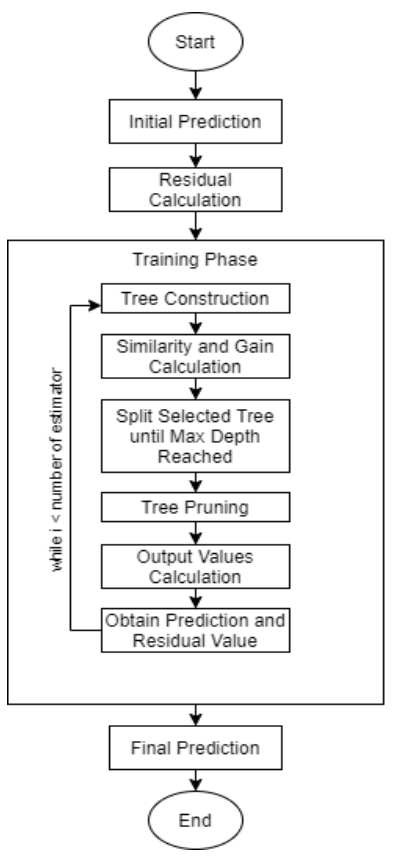

Figure 1 - XGBoost Process

\section{RESULT \& DISCUSSION}

\section{A. Long Short Term Memory (LSTM)}

After performing hyper parameter tuning on the LSTM model, the LSTM model shows a decrease in performance when making predictions with the addition of other countries' currency pairs. From the parameters used, it can be seen that shifting does not have much effect on improving performance of the model. This is concluded from the best results, where most of the best results use only 1 shifting Meanwhile, the number of layers, batch size and data length can be seen as having an influence on performance of the model. This is shown in the best results, where most of the best models have different combinations of parameters. The best LSTM model has 0.003197868 RMSE and is obtained when using a single dataset of USD/EUR currency pair with 3 LSTM layers, 1 shifting, batch size of 64 , and data length of 5 years $(2015$ $-2020)$.
TABle 3.

TOP 5 BEST RESULTS OF HYPER PARAMETER TUNING USING USD/EUR DATASET ON LSTM MODEL

\begin{tabular}{|cc|cc|c|c}
\hline No & \multicolumn{1}{c|}{ Layers } & Shifting & Batch Size & Data Length & RMSE \\
\hline 1 & {$[128,64,32]$} & 1 & 64 & 5 Years & 0.003197868 \\
\hline 2 & {$[128,64]$} & 1 & 64 & 5 Years & 0.003199347 \\
\hline 3 & {$[128]$} & 1 & 64 & 10 Years & 0.003268283 \\
\hline 4 & {$[128,64,32]$} & 1 & 64 & 10 Years & 0.003298315 \\
\hline 5 & {$[128,64]$} & 1 & 32 & 5 Years & 0.00329882 \\
\hline
\end{tabular}

TABLE 4.

TOP 5 BEST RESULTS OF HYPER PARAMETER TUNING USING USD/EUR, USD/GBP AND USD/JPY DATASET ON LSTM MODEL

\begin{tabular}{|c|l|c|c|c|c|}
\hline No & \multicolumn{1}{|c|}{ Layers } & Shifting & Batch Size & Data Length & RMSE \\
\hline 1 & {$[128]$} & 1 & 32 & 5 Years & 0.003290441 \\
\hline 2 & {$[128]$} & 1 & 64 & 5 Years & 0.003356335 \\
\hline 3 & {$[128,64,32]$} & 1 & 64 & 5 Years & 0.003450222 \\
\hline 4 & {$[128,64,32]$} & 1 & 64 & 10 Years & 0.003479094 \\
\hline 5 & {$[128]$} & 1 & 64 & 1 Years & 0.003479676 \\
\hline
\end{tabular}

TABLE 5.

TOP 5 BEST RESULTS OF HYPER PARAMETER TUNING USING USD/GBP AND USD/JPY DATASET ON LSTM MODEL

\begin{tabular}{|c|l|c|c|c|c|}
\hline No & \multicolumn{1}{|c|}{ Layers } & Shifting & Batch Size & Data Length & RMSE \\
\hline 1 & {$[128]$} & 1 & 16 & 10 Years & 0.005198272 \\
\hline 2 & {$[128,64]$} & 1 & 64 & 10 Years & 0.005493827 \\
\hline 3 & {$[128,64,32]$} & 1 & 64 & 10 Ycars & 0.005560829 \\
\hline 4 & {$[128]$} & 2 & 16 & 10 Years & 0.005575689 \\
\hline 5 & {$[128,64]$} & 1 & 16 & 10 Years & 0.005583348 \\
\hline
\end{tabular}

\section{B. Gated Recurrent Unit (GRU)}

After performing hyper parameter tuning on the GRU model, the GRU model shows a decrease in performance when making predictions with the addition of other countries currency pairs. From the parameters used, it can be seen that shifting does not have much effect on improving performance of the model. 


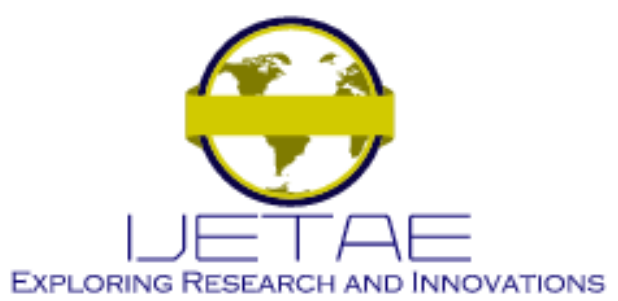

International Journal of Emerging Technology and Advanced Engineering Website: www.ijetae.com (E-ISSN 2250-2459, Scopus Indexed, ISO 9001:2008 Certified Journal, Volume 12, Issue 01, January 2022)

This is concluded from the best results, where most of the best results use only 1 shifting. Meanwhile, the number of layers, batch size and data length can be seen having an influence on performance of the model. This is shown in the best results, where most of the best models have different combinations of parameters. The best GRU model has $0.003234245 \mathrm{RMSE}$ and is obtained when using a single dataset of USD/EUR currency pair with 2 GRU layers, 1 shifting, batch size of 64 , and data length of 5 years $(2015-2020)$.

TABLE 6.

TOP 5 BEST RESULTS OF HYPER PARAMETER TUNING USING USD/EUR DATASET ON GRU MODEL

\begin{tabular}{|c|l|cc|c|c}
\hline No & \multicolumn{1}{|c|}{ Layers } & Shifting & Batch Size & Data Length & RMSE \\
\hline 1 & {$[128,64]$} & 1 & 64 & 5 Years & 0.003234245 \\
\hline 2 & {$[128,64,32]$} & 1 & 64 & 5 Years & 0.003234292 \\
\hline 3 & {$[128]$} & 1 & 64 & 5 Years & 0.003260869 \\
\hline 4 & {$[128,64,32]$} & 1 & 32 & 5 Years & 0.003277775 \\
\hline 5 & {$[128,64]$} & 1 & 32 & 5 Years & 0.003297893
\end{tabular}

TABLE 7.

TOP 5 BEST RESULTS OF HYPER PARAMETER TUNING USING USD/EUR, USD/GBP AND USD/JPY DATASET ON GRU MODEL

\begin{tabular}{c|l|c|c|c|c}
\hline No & \multicolumn{1}{|c|}{ Layers } & Shifting & Batch Size & Data Length & RMSE \\
\hline 1 & $\lceil 128\rceil$ & 1 & 64 & 10 Years & 0.003384393 \\
\hline 2 & $\lfloor 128\rfloor$ & 1 & 32 & 10 Years & 0.003468052 \\
\hline 3 & $\lceil 128\rceil$ & 1 & 32 & 5 Years & 0.003541196 \\
\hline 4 & $\lfloor 128\rfloor$ & 1 & 16 & 10 Years & 0.003552895 \\
\hline 5 & $\lfloor 128\rfloor$ & 1 & 64 & 1 Years & 0.003611328 \\
\hline
\end{tabular}

TABLE 8.

TOP 5 BEST RESULTS OF HYPER PARAMETER TUNING USING USD/GBP AND USD/JPY DATASET ON GRU MODEL

\begin{tabular}{|c|l|c|c|c|c|}
\hline No & \multicolumn{1}{|c|}{ Layers } & Shifting & Batch Size & Data Length & RMSE \\
\hline 1 & {$[128]$} & 1 & 16 & 10 Years & 0.005081361 \\
\hline 2 & {$[128,64]$} & 1 & 64 & 10 Years & 0.005450542 \\
\hline 3 & {$[128]$} & 2 & 16 & 10 Years & 0.005450805 \\
\hline 4 & {$[128,64]$} & 1 & 16 & 10 Years & 0.005471086 \\
\hline 5 & {$[128,64,32]$} & 1 & 64 & 10 Years & 0.005725326 \\
\hline
\end{tabular}

\section{Support Vector Regression (SVR)}

After performing hyper parameter tuning on the SVR model, the SVR model shows a slight increase in performance when making predictions with the addition of other countries's currency pairs. From the parameters used, it can be seen that shifting does not have much effect on improving performance of the model. This is concluded from the best results, where most of the best results use only 1 shifting. Meanwhile, the kernel, regularization, gamma and data length can be seen having an influence on performance of the model. This is shown in the best results, where most of the best models have different combinations of parameters. The best SVR model has 0.003432971 and is obtained when using combination dataset of USD/EUR, USD/GBP and USD/JPY currency pairs with sigmoid kernel, regularization of 100, 1 shifting, auto gamma and data length of 10 years (2010-2020).

TABLE 9.

TOP 5 BEST RESULTS OF HYPER PARAMETER TUNING USING USD/EUR DATASET ON SVR MODEL

\begin{tabular}{|c|ll|c|c|c|c|}
\hline No & Kernel & Regularization & Shifting & Gamma & $\begin{array}{c}\text { Data } \\
\text { Length }\end{array}$ & RMSE \\
\hline 1 & rbf & 100 & 1 & 0.1 & 5 Years & 0.003442543 \\
\hline 2 & rbf & 100 & 1 & 0.2 & 5 Years & 0.00344803 \\
\hline 3 & rbf & 100 & 1 & auto & 5 Years & 0.00344803 \\
\hline 4 & linear & 100 & 1 & 0.1 & 5 Years & 0.003464667 \\
\hline 5 & linear & 100 & 1 & 0.2 & 5 Years & 0.003464667 \\
\hline
\end{tabular}

TABLE 10.

TOP 5 BEST RESULTS OF HYPER PARAMETER TUNING USING USD/EUR, USD/GBP AND USD/JPY DATASET ON SVR MODEL

\begin{tabular}{|c|ll|c|c|c|c|}
\hline No & Kernel & Regularization & Shifling & Gamma & $\begin{array}{c}\text { Data } \\
\text { Length }\end{array}$ & RMSE \\
\hline 1 & sigmoid & 100 & 1 & auto & 10 Years & 0.003432971 \\
\hline 2 & sigmoid & 1000 & 1 & auto & 10 Years & 0.003432971 \\
\hline 3 & sigmoid & 10000 & 1 & auto & 10 Years & 0.003432971 \\
\hline 4 & rbf & 100 & 1 & auto & 5 Years & 0.003655812 \\
\hline 5 & rbf & 100 & 1 & auto & 10 Years & 0.003662809 \\
\hline
\end{tabular}




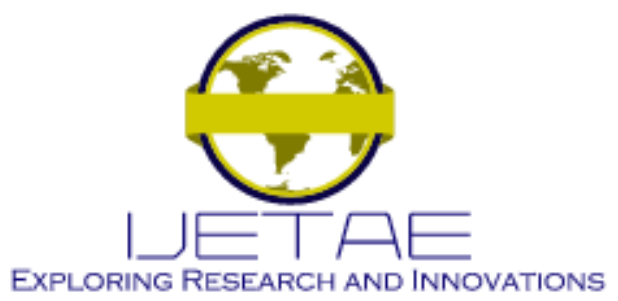

International Journal of Emerging Technology and Advanced Engineering

Website: www.ijetae.com (E-ISSN 2250-2459, Scopus Indexed, ISO 9001:2008 Certified Journal, Volume 12, Issue 01, January 2022)

TABLE 11.

TOP 5 BEST RESULTS OF HYPER PARAMETER TUNING USING USD/GBP AND USD/JPY DATASET ON SVR MODEL

\begin{tabular}{|c|ll|c|c|cc|}
\hline No & Kerncl & Regularization & Shifting & Gamma & $\begin{array}{c}\text { Data } \\
\text { Length }\end{array}$ & RMSE \\
\hline 1 & rbf & 100 & 1 & auto & 10 Years & 0.008496377 \\
\hline 2 & rbf & 100 & 1 & 0.1 & 10 Years & 0.008626839 \\
\hline 3 & poly & 100 & 1 & auto & 10 Years & 0.008938521 \\
\hline 4 & linear & 10000 & 1 & 0.1 & 10 Years & 0.008998083 \\
\hline 5 & linear & 10000 & 1 & 0.2 & 10 Years & 0.008998083 \\
\hline
\end{tabular}

D. Auto Regressive Integrated Moving Average (ARIMA)

Prediction using ARIMA is only done on the USD / EUR dataset. The procedure for making predictions is divided into 2 methods, prediction without retraining the model and prediction by retraining the model. Prediction without re-training the model is done by only doing 1 training phase then making predictions for the next $n$-days. Whereas, prediction by retraining the model is carried out by continuously adding the actual value during the train then making predictions for the next 1 day which will then be stored into the array. To get the best results, hyper parameter tuning is also applied by combining several parameters such as d, q and p values, data shifting, and the length of the dataset. The prediction results using ARIMA show a significant increase in performance when the model is retrained every time it makes predictions with the best RMSE value of 0.003109 . More detailed results can be seen in table 11 and table 12 .

TABLE 12.

TOP 5 BEST RESULTS OF HYPER PARAMETER TUNING USING DATASETS USD/EUR WITH RETRAINING ARIMA MODEL

\begin{tabular}{|c|c|c|c|c|c|c|}
\hline No & $\mathrm{p}$ & $\mathrm{d}$ & $\mathrm{q}$ & Shifting & Data Length & RMSE \\
\hline 1. & 4 & 2 & 2 & 2 & 10 years & 0.003109781558 \\
\hline 2. & 4 & 2 & 1 & 2 & 10 years & 0.003126651757 \\
\hline 3. & 4 & 1 & 1 & 2 & 10 years & 0.003127698184 \\
\hline 4. & 3 & 1 & 1 & 2 & 10 years & 0.003153035643 \\
\hline 5. & 3 & 1 & 2 & 2 & 10 years & 0.003159656975 \\
\hline
\end{tabular}

TABLE 13.

TOP 5 BEST RESULTS OF HYPER PARAMETER TUNING USING DATASETS USD/EUR WITHOUT RETRAINING ARIMA MODEL

\begin{tabular}{|c|c|c|c|c|c|c|}
\hline No & $\mathrm{p}$ & $\mathrm{d}$ & $\mathrm{q}$ & Shifting & Data Length & RMSE \\
\hline 1. & 3 & 1 & 2 & 10 & 10 years & 0.02661928681 \\
\hline 2. & 4 & 1 & 4 & 10 & 10 years & 0.02662843678 \\
\hline 3. & 5 & 1 & 1 & 10 & 10 years & 0.02664229106 \\
\hline 4. & 5 & 1 & 3 & 10 & 10 years & 0.02664408128 \\
\hline 5. & 4 & 1 & 4 & 10 & 10 years & 0.02665651036 \\
\hline
\end{tabular}

\section{E. XGBoost}

To get the best results for this model, the hyper parameter tuning method will be carried out for the parameters: estimator, shifting, data length and type of objective function. After performing hyper parameter tuning on the XGBOOST model, the best results were obtained in the combined dataset of USD / EUR, USD / GBP and USD / JPY with an RMSE value of 0.003098 . The XGBOOST model shows an increase in performance when making predictions with the addition of datasets for other countries' currencies. From the parameters used, it can be seen that an increase in the number of estimators does not have much effect on the resulting RMSE. Meanwhile, the most influential parameters are the amount of shifting and the length of the dataset used for training. The best results are obtained by shifting the data once and using data from 2010 - 2020 (10 years dataset). More detailed results can be seen in table 13, table 14 and table 15.

TABLE 14.

TOP 5 BEST RESULTS OF HYPER PARAMETER TUNING USING DATASETS USD/EUR ON XGBOOST MODEL

\begin{tabular}{|c|c|c|c|l|c|}
\hline No & Estimator & Shifting & Data Length & Objective Function & RMSE \\
\hline 1 & 100 & 1 & 10 years & reg:squaredlogerror & 0.003239133917 \\
\hline 2 & 400 & 1 & 10 years & reg:squaredlogerror & 0.003253752974 \\
\hline 3 & 800 & 1 & 10 years & reg:squaredlogerror & 0.003253753434 \\
\hline 4 & 1600 & 1 & 10 years & reg:squaredlogerror & 0.003253753434 \\
\hline 5 & 3200 & 1 & 10 years & reg:squaredlogerror & 0.003253753434 \\
\hline
\end{tabular}




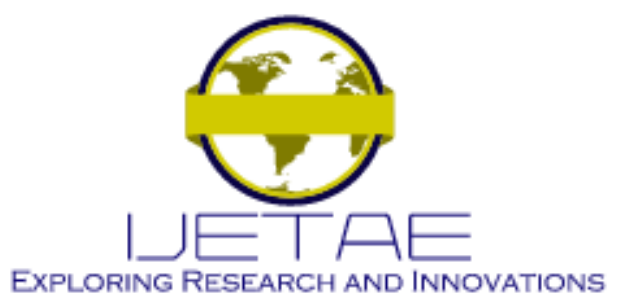

International Journal of Emerging Technology and Advanced Engineering

Website: www.ijetae.com (E-ISSN 2250-2459, Scopus Indexed, ISO 9001:2008 Certified Journal, Volume 12, Issue 01, January 2022)

TABLE 15.

TOP 5 BEST RESULTS OF HYPER PARAMETER TUNING USING DATASETS USD/EUR, USD/GBP AND USD/JPY ON XGBOOST MODEL

\begin{tabular}{|c|c|c|c|c|c|}
\hline No & Estimator & Shifting & Data Length & Objective Function & RMSE \\
\hline 1 & 200 & 1 & 10 years & reg:squarederror & 0.003098305151 \\
\hline 2 & 400 & 1 & 10 years & reg:squarederror & 0.003098306149 \\
\hline 3 & 800 & 1 & 10 years & reg:squarederror & 0.003098307231 \\
\hline 4 & 1600 & 1 & 10 years & reg:squarederror & 0.003098308061 \\
\hline 5 & 3200 & 1 & 10 years & reg:squarederror & 0.003098308061 \\
\hline
\end{tabular}

TABLE 16.

TOP 5 BEST RESULTS OF HYPER PARAMETER TUNING USING DATASETS USD/GBP AND USD/JPY ON XGBOOST MODEL

\begin{tabular}{|c|c|c|c|l|c|}
\hline No & Estimator & Shifting & Data Length & Objective Function & RMSE \\
\hline 1 & 200 & 1 & 10 years & reg:squarederror & 0.006050351083 \\
\hline 2 & 400 & 1 & 10 years & reg:squarederror & 0.006050351083 \\
\hline 3 & 800 & 1 & 10 years & reg:squarederror & 0.006071953269 \\
\hline 4 & 1600 & 1 & 10 years & reg:squarederror & 0.006071953269 \\
\hline 5 & 3200 & 1 & 10 years & reg:squarederror & 0.006071953795 \\
\hline
\end{tabular}

\section{F. Summary}

From all the available results, the model with the best RMSE value is the XGBOOST model using the USD / EUR, USD / GBP, and USD / JPY dataset with a RMSE value of 0.003098305151 . A detailed summary of the RMSE results from each model are shown in table 16. (Note : RMSE of LSTM and GRU is average of each RMSEs after 10 loop).
TABLE 17.

SUMMARY RMSE RESULT FROM EACH MODEL

\begin{tabular}{|c|c|}
\hline Model & RMSE \\
\hline XGBOOST USD/EUR, USD/GBP, USD/JPY & 0.003098305151 \\
\hline ARIMA with retrainUSD/EUR & 0.003109781558 \\
\hline XGBOOST USD/EUR & 0.003239133917 \\
\hline GRU USD/EUR & 0.003242897727 \\
\hline LSTM USD/EUR & 0.003292500883 \\
\hline LSTM USD/EUR, USD/GBP, USD/JPY & 0.003367967893 \\
\hline SVR USD/EUR, USD/GBP, USD/JPY & 0.003432971488 \\
\hline SVR USD/EUR & 0.003442543136 \\
\hline GRU USD/EUR, USD/GBP, USD/JPY & 0.003772032 \\
\hline GRU USD/GBP, USD/JPY & 0.005064127515 \\
\hline LSTM USD/GBP, USD/JPY & 0.005124483546 \\
\hline XGBOOST USD/GBP, USD/JPY & 0.006050351083 \\
\hline SVR USD/GBP, USD/JPY & 0.008496376779 \\
\hline ARIMA without retrain USD/EUR & 0.02661928681 \\
\hline
\end{tabular}

\section{CONCLUSION}

Exchange rates on Forex markets are always changing and it is hard to predict. The main problem is the uncertainty that affects the ups and downs of what is being predicted. Uncertainty that affects currency exchange rate movements can be in the form of inflation rates, interest rates, government debt, terms of trade, political stability of certain countries, recession and many more. This uncertainty can be reduced with the help of technology using machine learning.

In this study, various machine learning models were used to predict forex data and analyze the effect of one forex on another. 


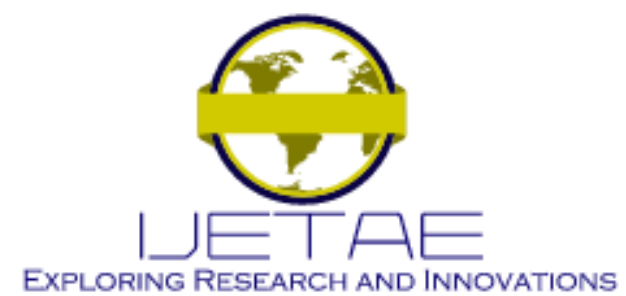

International Journal of Emerging Technology and Advanced Engineering Website: www.ijetae.com (E-ISSN 2250-2459, Scopus Indexed, ISO 9001:2008 Certified Journal, Volume 12, Issue 01, January 2022)

Machine learning models used in this research are LSTM, GRU, SVR, XGBOOST and ARIMA. XGBOOST ends up handling the data the best with an RMSE value of 0.00309830 by using USD/EUR, USD/GBP, USD/JPY dataset. The obtained results show that external data can improve the performance of the prediction results. However, the performance improvement using external datasets only applies to certain models such as the XGBOOST model in this study.

\section{REFERENCES}

[1] Tiong LCO, Ngo DCL, Lee Y. Forex prediction engine: Framework, modelling techniques and implementations. Int J Comput Sci Eng 2016;13:364-77. https://doi.org/10.1504/IJCSE.2016.080213.

[2] Kokra VS. Analysis of Stock Price Prediction using CNN model based on the Historical Stock Prices. Int J Sci Eng Res 2020;11:85461.

[3] Lu W, Li J, Li Y, Sun A, Wang J. A CNN-LSTM-based model to forecast stock prices. Complexity 2020;2020. https://doi.org/10.1155/2020/6622927.

[4] Kwon YK, Sun H Di. A hybrid system integrating a piecewise linear representation and a neural network for stock prediction. Proc. 6th Int. Forum Strateg. Technol. IFOST 2011, vol. 2, 2011, p. 796-9. https://doi.org/10.1109/IFOST.2011.6021141.

[5] Rahul Mangalampalli, Pawankumar Khetre, Vaibhav Malviya, Vivek Pandey. Stock Prediction using Hybrid ARIMA and GRU Models. Int J Eng Res 2020;V9:737-43. https://doi.org/10.17577/ijertv9is050550.

[6] Hegazy O, Soliman OS, Salam MA. A Machine Learning Model for Stock Market Prediction. Int J Comput Sci Telecommun 2014;4:1723.

[7] Kia AN, Fathian M, Gholamian MR. Using MLP and RBF Neural Networks to Improve thePrediction of Exchange Rate Time Series with ARIMA. Int J Inf Electron Eng 2012;3:2-6. https://doi.org/10.7763/ijiee.2012.v2.157.

[8] Xiao C, Xia W, Jiang J. Stock price forecast based on combined model of ARI-MA-LS-SVM. Neural Comput Appl 2020;32:537988. https://doi.org/10.1007/s00521-019-04698-5.

[9] Banik S, Khodadad Khan AFM, Anwer M. Hybrid machine learning technique for forecasting dhaka stock market timing decisions. Comput Intell Neurosci 2014;2014. https://doi.org/10.1155/2014/318524.

[10] Beneki C, Yarmohammadi M. Forecasting exchange rates: An optimal approach. J Syst Sci Complex 2014;27:21-8. https://doi.org/10.1007/s11424-014-3304-5.

[11] Yu L, Wang S, Lai KK. A novel nonlinear ensemble forecasting model incorporating GLAR and ANN for foreign exchange rates. Comput Oper Res 2005;32:2523-41. https://doi.org/10.1016/j.cor.2004.06.024.
[12] Saiful Islam M, Hossain E. Foreign Exchange Currency Rate Prediction using a GRU-LSTM Hybrid Network. Soft Comput Lett 2020:1-19. https://doi.org/10.1016/j.socl.2020.100009.

[13] Investing.com n.d. https://www.investing.com.

[14] USD/EUR - US Dollar Euro n.d. https://www.investing.com/currencies/usd-eur-historical-data.

[15] USD/GBP - US Dollar British Pound n.d. https://www.investing.com/currencies/usd-gbp-historical-data.

[16] USD/JPY - US Dollar Japanese Yen n.d.

[17] Hochreiter S, Schmidhuber J. Long Short-Term Memory. Neural Comput 1997:9:1735-80. https://doi.org/10.1162/neco.1997.9.8.1735.

[18] Kim HY, Won CH. Forecasting the volatility of stock price index: A hybrid model integrating LSTM with multiple GARCH-type models. Expert Syst Appl 2018;103:25-37. https://doi.org/10.1016/j.eswa.2018.03.002.

[19] Greff K, Srivastava RK, Koutnik J, Steunebrink BR, Schmidhuber J. LSTM: A Search Space Odyssey. IEEE Trans Neural Networks Learn Syst 2017;28:2222-32. https://doi.org/10.1109/TNNLS.2016.2582924.

[20] Cho K, Van Merriënboer B, Gulcehre C, Bahdanau D, Bougares F, Schwenk $\mathrm{H}$, et al. Learning phrase representations using RNN encoder-decoder for statistical machine translation. EMNLP 2014 2014 Conf. Empir. Methods Nat. Lang. Process. Proc. Conf., Doha, Qatar: 2014, p. 1724-34. https://doi.org/10.3115/v1/d14-1179.

[21] Gao Y, Glowacka D. Deep gate recurrent neural network. Work. Conf. Proc., vol. 63, Hamilton: The Journal of Machine Learning Research; 2016, p. 350-65.

[22] Li L, Hu C, Hou Y. Prediction analysis of shield vertical attitude based on GRU. J. Phys. Conf. Ser., vol. 1651, Guilin: IOP Publishing; 2020. https://doi.org/10.1088/1742-6596/1651/1/012032.

[23] Lingras P, Butz CJ. Rough support vector regression. Eur J Oper Res 2010;206:445-55. https://doi.org/10.1016/j.ejor.2009.10.023.

[24] Wibowo A. Optimization for Cutting Conditions of Surface Roughness in Machining Process Using Support Vector Regression and Genetic Algorithm. Int J Mech Mechatronics Eng IJMMEIJENS 2019;19:25-34.

[25] Parveen N, Zaidi S, Danish M. Support Vector Regression Prediction and Analysis of the Copper (II) Biosorption Efficiency. Indian Chem Eng 2017;59:295-311. https://doi.org/10.1080/00194506.2016.1270778.

[26] Shahriar SA, Kayes I, Hasan K, Hasan M, Islam R, Awang NR, et al. Potential of arima-ann, arima-svm, dt and catboost for atmospheric pm2.5 forecasting in bangladesh. Atmosphere (Basel) 2021;12:1-21. https://doi.org/10.3390/atmos12010100.

[27] Brownlee J. A Gentle Introduction to XGBoost for Applied Machine Learning 2016. 